\title{
Apparatus for the Detection of Piezoelectric Coupling
}

\author{
Lothar Frenkel
}

(February 1, 1963)

\begin{abstract}
An apparatus for the investigation of the piezoelectric coupling in granular powders is described. The apparatus is based on the method of Giebe and Scheibe but employs the sensitive detection and recording methods commonly employed in the study of nuclear quadrupole resonances. Some sample recorder traces are shown. The traces indicate that the apparatus is a workable qualitative tool with at least the possibility of somequantitative usefulness.7
\end{abstract}

\section{Apparatus for the Detection of Piezo- electric Coupling}

The standard method used for the qualitative determination of the presence of piezoelectric coupling in small crystals is that of Giebe and Scheibe $[1,2,3] .{ }^{1}$ In this method a sensitive oscillator is tuned over a frequency range in the short wave band. A small test capacitor is filled with the sample and put in parallel with the tuning condenser.

When the oscillator frequency passes the mechanical resonance frequency of a grain of the material under test the grain is momentarily excited by piezoelectric coupling to the oscillating field and energy is absorbed by the grain from the oscillator. The oscillator output is monitored by a detector and a pair of earphones which register the resonances as clicks. This method of detecting is rather cumbersome, of limited sensitivity and inherently difficult to use quantitatively.

In recent years interest in radiofrequency spectroscopy has led to a number of simple and sensitive instruments for the detection and measurement of minute absorptions of radiofrequency energies which could very well be used for the study of weakly piezoelectric substances.

Livingston [4] noted that the apparatus used by him to study the pure nuclear quadrupole resonance of $\mathrm{Cl}$ in sodium chlorate registered spurious signals at various frequencies which he interpreted as piezoelectric responses of grains of the substance under study in the coil of the oscillator. The stray electric fields across the coil of his oscillator presumably caused the response of the grains.

1 Figures in brackets indicate the literature references at the end of this paper.
Having observed this effect independently during a study of nuclear quadrupole resonances, we proceeded to investigate the response of our equipment to grains placed, as in the method of Giebe and Scheibe, in a small capacitor. We found that this combination of the Giebe and Scheibe method with techniques commonly employed in nuclear resonance studies does indeed result in a very sensitive detector for piezoelectric coupling.

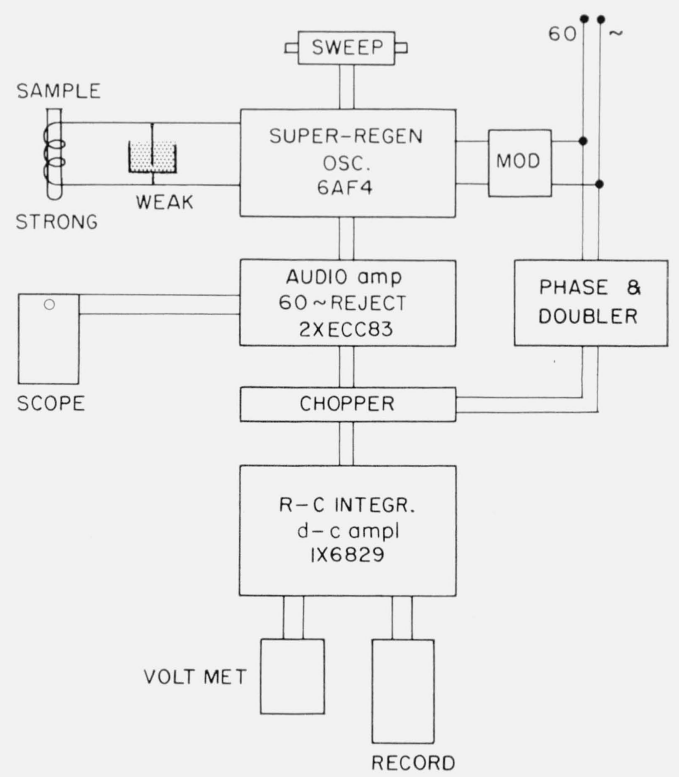

Figure 1. Block diagram of apparatus. 


\section{Descriptions of Apparatus}

A block diagram of our apparatus is given in figure 1. The oscillator, a $6 \mathrm{AF} 4$ triode, is arranged as a superregenerative detector with a quench frequency of approximately $30 \mathrm{kc} / \mathrm{s}$. Three individual capacitors make up the capacitance in the tank circuit. Their functions are as follows:

(1) A variable tuning capacitor driven by a small clock motor allows the center frequency to be swept slowly over a range of about $1 \mathrm{Mc} / \mathrm{s}$ in the vicinity of $25 \mathrm{Mc} / \mathrm{s}$; (2) a vibrating capacitor made out of a small loudspeaker modulates the frequency of the oscillator by a maximum of about $10 \mathrm{kc} / \mathrm{s}$ from the instantaneous center frequency. The modulating frequency is $60 \mathrm{c} / \mathrm{s}$.; (3) finally, a small test capacitor (shown in fig. 2) holds the sample under test.

If the center frequency of the oscillator coincides with the mechanical resonance frequency of a grain the oscillator will be loaded twice in every complete cycle of modulation; i.e., each time the modulation amplitude is zero. This results in a signal of twice the modulation frequency at the oscillator plate. This signal is filtered and amplified in a four-stage low noise audio amplifier. To minimize noise the signal is then chopped at twice the modulation frequency and the chopped signal is integrated in an RC network with a long time constant. The output from the integrating capacitor may be displayed on a recorder after suitable amplification.

We have made no attempt to analyze the recorder responses theoretically. Clearly the signal seen on the recorder as the frequency of the oscillator is slowly swept depends on a large number of variables such as the quench frequency of the superregenerative detector, the modulation amplitude, the chopper phase as well as on the number and shape of the grains in the sample. It should be noted that the

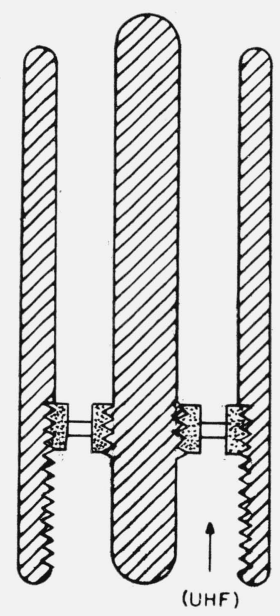

Figure 2. Sample holder. response in this device does not result from individual grains as in the methods of Giebe and Scheibe, but rather from the random number of grains having their mechanical resonances near the instantaneous center frequency. This is because the modulation frequency is much larger than the width of each grain's mechanical resonance response. The signal to sample size relationship therefore is that of random fluctuations.

\section{Results}

To test the apparatus, a number of substances listed in reference 2 as having weak or very weak coupling were tried and the resulting tracks are shown in figure 3 . Substances for which the cou-
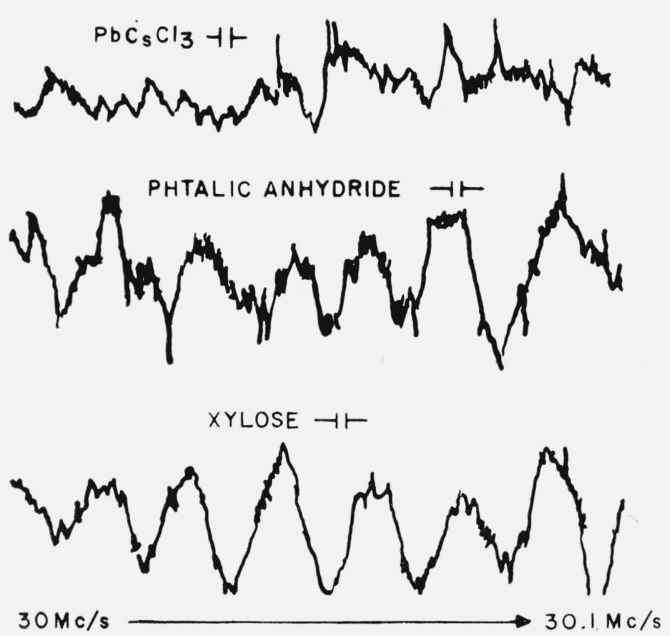

FiguRE 3a. Recorder responses versus frequency for substances placed in the test capacitor.

The top trace in figure $3 \mathrm{~b}$ shows the background.
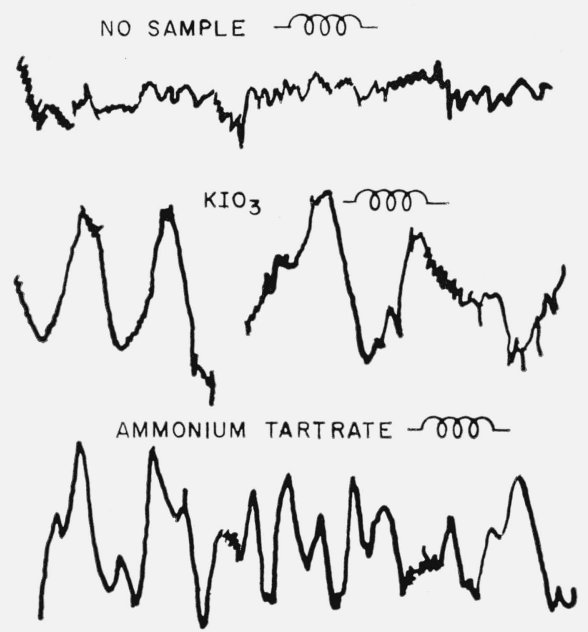

$30 \mathrm{Mc} / \mathrm{s}$ $39.1 \mathrm{Mc} / \mathrm{s}$

Figure 3b. Recorder response versus frequency with material in the coil of the oscillator. 

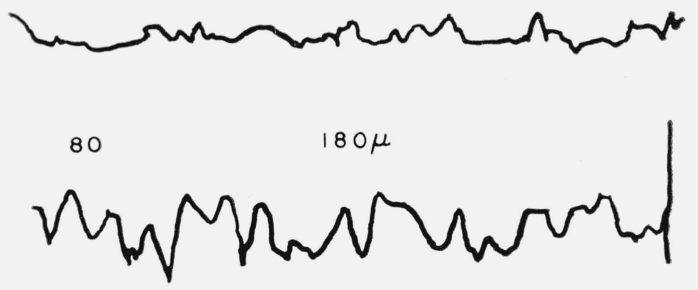

140

$100 \mu$

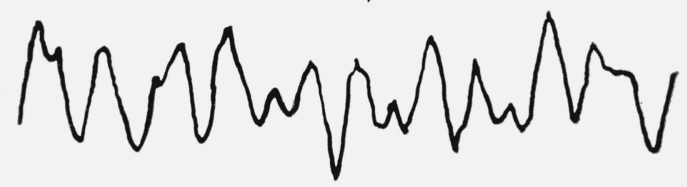

170

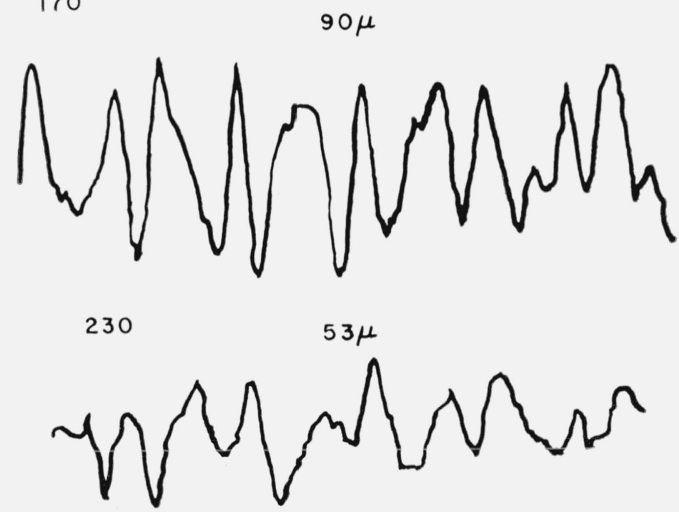

io $\mathrm{Mc} / \mathrm{s}$ $30.1 \mathrm{Mc} / \mathrm{s}$

FigURE 4. Recorder response versus frequency as a function of grain size for $\mathrm{NaClO}_{3}$.

The numbers on the right are sieve numbers. pling is very strong as seen by this device are best tested in the coil since the strong signals otherwise saturate the amplifier. The traces for potassium iodate and ammonium tartrate in figure 3 were obtained in this way.

For other materials, in particular barium nitrate and dibasic ammonium citrate, no tracks were seen.

Potassium iodate is listed by Mason as having no coupling. In this material phase changes were first discovered by Hettich, [5] using the method of Giebe and Scheibe, and, more recently, both the piezoelectric and nuclear quadrupole characteristics of this material were investigated by Herlach, [6] who also discovered its ferro-electric properties.

For such work the method described here would seem ideal, first, because nuclear quadrupole equipment is available and can be used with minor changes for the investigation of piezoelectric coupling and secondly because the recorder traces may be evaluated in a semiquantitative way as regards the strength of the coupling. Figure 4, for instance, shows the effect of selecting grain size at equal settings.

The author is indebted to M. Greenspan and L. Bennett for advice, and to Van Johnson for suggestions concerning the electronics.

\section{References}

[1] E. Giebe and A. Scheibe, ZS f. Phys. 33, 760 (1925).

[2] Mason, Piezoelectric crystals and their applications to Ultrasonics, pp. 47-58 (D. van Nostrand, 1950).

[3] S. B. Elings and P. Terpstra, Z. Krist. 67, 279 (1928).

4] Livingston Ann. N.Y. Acad. of Sciences 55, 800 (1952).

[5] A. Hettich, ZS fur Phys. 65, 506 (1930).

[6] F. Herlach, Helv. Phys. Acta 34, 305-330 (1961).

(Paper 67C3-129) 